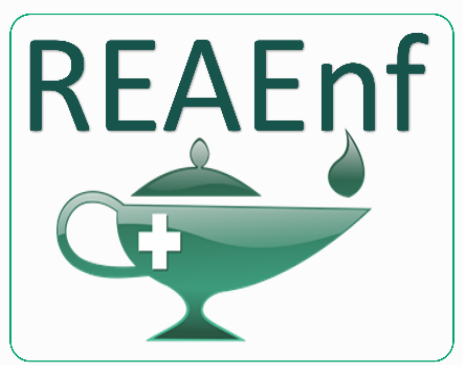

Revista Eletrônica Acervo Enfermagem

\section{RELATO DE EXPERIÊNCIA}

Recebido em: 10/2020

Aceito em: $11 / 2020$

Publicado em: 12/2020

\title{
Vivência de estudantes de enfermagem como monitores na disciplina de semiologia e semiotécnica
}

\author{
Experience of nursing students as monitors in the discipline of semiology and \\ semiotechnics
}

Experiencia de estudiantes de enfermería como monitores en la disciplina de semiología y semiotecnica

Italo Everton Bezerra Barbosa ${ }^{1 *}$, Alicia Ribeiro Fonseca ${ }^{1}$, Meire Cristina Soares Ribeiro.

\begin{abstract}
Resumo: Esse artigo buscou descrever as experiências vivenciadas no programa de monitoria acadêmica na disciplina de semiologia e semiotécnica, com os alunos do quarto período, do turno matutino no curso de Bacharelado em Enfermagem de um centro universitário de Manaus, utilizando o método hipotético-dedutivo, em conjunto com o tipo de pesquisa descritiva. Trata-se de um relato de experiência, vivenciado por dois acadêmicos de enfermagem na disciplina semiologia e semiotécnica oferecido por uma universidade particular localizado na capital do estado do Amazonas. No primeiro momento, as aulas teóricas estavam direcionadas principalmente em dar auxílio ao professor em sala, desenvolvendo diversas atividades como auxiliar na aplicação e correção das provas. Entretanto, no segundo momento as aulas práticas foram direcionadas principalmente a realização de práticas laboratoriais como aspiração das vias aéreas, técnicas de sondagem enteral, gástrica, aplicação de medicamentos no deltoide, vasto lateral, dorso glúteo. Desse modo, a monitoria configurou-se como uma importante atividade exercida pelo monitor, pois possibilitou uma nova oportunidade na vida acadêmica tanto pela possibilidade de rever os conteúdos ministrados em aula como também para adquirir novos conhecimentos.
\end{abstract}

Palavras-chave: Semiologia, Semiotécnica, Monitoria.

\begin{abstract}
This article sought to describe the experiences lived in the academic monitoring program in the discipline of semiology and semiotechnology, with the students of the fourth period, of the morning shift in the Bachelor of Nursing course of a university center in Manaus, using the hypothetical-deductive method, together with the type of descriptive research, it is an experience report, experienced by two nursing students in the semiotics and semiotics discipline offered by a private university located in the capital of the state of Amazonas. In the first moment, the theoretical classes were mainly aimed at assisting the teacher in the classroom, developing various activities as an aid in the application and correction of tests. However, in the second moment, the practical classes were mainly directed to the realization of laboratory practices such as aspiration of the airways, enteral, gastric probing techniques, application of drugs in the deltoid, vastus lateralis, buttocks. Thus, the monitoring was configured as an important activity performed by the monitor, as it enabled a new opportunity in academic life, both because of the possibility of reviewing the contents taught in class and also to acquire new knowledge.
\end{abstract}

Keywords: Semiology, Semiotechnique, Monitoring.

${ }^{1}$ Centro Universitário Fametro (FAMETRO), Manaus - AM.

*E-mail: italoeverton1998@gmail.com 
Resumen: Este artículo buscó describir las experiencias vividas en el programa de seguimiento académico en la disciplina de semiología y semiotecnología, con los estudiantes del cuarto período, del turno matutino de la carrera de Licenciatura en Enfermería de un centro universitario en Manaus, utilizando el método hipotético-deductivo, junto con el tipo de investigación descriptiva, es un relato de experiencia, vivido por dos estudiantes de enfermería en la disciplina de semiótica y semiótica que ofrece una universidad privada ubicada en la capital del estado de Amazonas. En un primer momento, las clases teóricas estuvieron orientadas principalmente a asistir al docente en el aula, desarrollando diversas actividades como ayuda en la aplicación y corrección de pruebas. Sin embargo, en el segundo momento, las clases prácticas se dirigieron principalmente a la realización de prácticas de laboratorio como aspiración de las vías respiratorias, técnicas de sondaje enteral, gástrico, aplicación de medicamentos en el deltoides, vasto lateral, dorso glúteo. Así, el seguimiento se configuró como una actividad importante realizada por el monitor, ya que posibilitó una nueva oportunidad en la vida académica, tanto por la posibilidad de revisar los contenidos impartidos en clase como por adquirir nuevos conocimientos.

Palabras clave: Semiología, Semiotécnica, Seguimiento.

\section{INTRODUÇÃO}

A disciplina de semiologia e semiotécnica visa garantir, que os alunos do curso de Bacharelado em Enfermagem obtenham conhecimento pela participação as aulas teóricas e práticas, baseadas em bibliografias, revisão de literaturas, e artigos publicados. Para isso, utilizam-se as Metodologias Ativas em diversas atividades acadêmicas, como planejamentos de gincanas, orientação aos alunos, mesas redondas e uso do laboratório que facilita o contato com peças anatômicas e cadáveres, enriquecendo mais o processo de ensino-aprendizagem dos alunos (SANTOS GM e BATISTA SH, 2015).

Dentre as Metodologias Ativas, destaca-se o programa de Monitoria, que inclui ao aluno uma formação integrada nas atividades de extensão e pesquisas realizadas dentro da instituição. O exercício da monitoria acadêmica é percebido como um subsídio necessário para prática do profissional como futuro docente, pois o aluno-monitor além de complementar seus conhecimentos adquiridos no decorrer da graduação, adquire novas habilidades, capacidade de interação e trabalha a postura diante de determinadas situações, seja ela envolvendo a vida profissional ou acadêmica (GUEDES HD, et al., 2018).

No decorrer da graduação é através da monitoria que começamos a ter novas oportunidades de crescimento acadêmico. Visto que, o exercício da monitoria possui como objetivo o desenvolvimento de novos paradigmas de ensino e aprendizagem. Desse modo, nota-se um alinhamento entre os interessados com as necessidades apresentadas nas atividades desempenhadas pelo monitor (SANTOS AR, 2015).

A monitoria acadêmica é entendida como um grande instrumento para a melhoria do ensino de graduação, pois através dela e estabelecido novas práticas e experiências pedagógicas que visem fortalecer a articulação entre a teoria e prática. Uma vez que, quanto mais o monitor vivência a condição de acadêmico na mesma disciplina, mais sensível torna-se na percepção de solucionar prováveis dificuldades que ele venha encontrar relacionado ao conteúdo, conseguindo auxiliar com maior facilidade o aluno na solução dos problemas. Essa dinâmica promove o estreitamento de vínculo com a realidade docente e o desenvolvimento das práticas pedagógicas (ALMEIDA RS, et al., 2016).

O aluno que possui o auxílio do monitor, consegue dispor de novas oportunidades de adquirir novos conhecimentos e compreender melhor o assunto abordado pelo professor, facilitando assim no esclarecimento de suas dúvidas quanto ao conteúdo programático da disciplina reforçando o aprendizado. Entretanto, existem alguns alunos que de certa forma negligenciam o suporte oferecido pelo monitor, e com isso acabam perdendo a troca de conhecimentos que seria gerada pelo aluno-monitor que já cursou aquela disciplina (STEINDORFF G, et al., 2016).

Desse modo, surge a monitoria acadêmica como uma ferramenta de apoio pedagógico, oferecido os discentes que estão envolvidos no processo acadêmico e interessados em se aprofundar nos conteúdos e solucionar as dificuldades que iram ser abordadas no decorrer em sala de aula, proporcionando um espaço de discussões e debates acerca das temáticas de cada disciplina (FERNANDES NC, et al., 2015).

REAEnf/EJNC | Vol. 6 | e5685 | DOI: https://doi.org/10.25248/REAenf.e5685.2020 Página 2 de 6 
Com o desenvolvimento das atividades de monitoria, o estudante tem a oportunidade de se tornar mais autônomo, confiante e capaz de se posicionar frente às situações e os problemas que possam surgir durante o processo de ensino-aprendizagem, assim como também estimula a construir novos projetos pessoais, estabelecer e seguir metas, buscar formas para superar suas dificuldades e as colocar em prática para tentar resolver as situações (FRISON LM, 2010).

A disciplina de Semiologia e Semiotécnica tem um papel extremamente importante na grade curricular do curso de bacharelado em enfermagem. Tendo em vista que, semiologia e a busca e o estudo dos sinais e sintomas que o paciente pode apresentar durante a coletados de dados na anamnese, já semiotécnica e o campo que abrange métodos, técnicas e procedimentos que serão utilizados nas ações que sucedem o exame físico, as possíveis intervenções para solucionar os problemas (MELO GSM, et al., 2016).

Desse modo, a monitoria se configura como uma estratégia de grande relevância para o processo de ensino/aprendizagem desses alunos. Visto que, ela proporciona aos estudantes desenvolverem as novas competências que estão envolvidas nesse processo, como a comunicação em público, o relacionamento interpessoal e principalmente a adaptação de atuação durante as práticas clínicas, seja envolvendo o ambiente hospitalar ou na atenção básica à saúde (GURGEL SS e TAVEIRA GP, et al., 2017).

Com isso, estes pontos citados e associado as ideias no texto de Cardoso MC e Araujo RP (2008), que descrevem sobre o programa de monitoria, fundamentam o presente Relato de Experiência que objetiva descrever as experiências vivenciadas no programa de monitoria acadêmica na disciplina de semiologia e Semiotécnica do curso de bacharelado em enfermagem.

Uma vez que, está disciplina disponibiliza ao aluno uma gama de novos conhecimentos sobre procedimentos e técnicas de enfermagem, algo que será de extrema importância para sua atuação como futuro enfermeiro.

\section{RELATO DE EXPERIÊNCIA}

Trata-se de um relato de experiência descritivo, vivenciado por dois acadêmicos de enfermagem na disciplina semiologia e semiotécnica oferecido por uma universidade particular localizado na capital do estado do Amazonas. Orientados pela professora Meire Cristina Soares Ribeiro, direcionada a Turma do quarto período, do turno matutino no curso de Bacharelado em Enfermagem de um Centro Universitário de Manaus.

Utilizou-se o método hipotético-dedutivo proposto por Popper, com o tipo de pesquisa descritiva, que visa descrever as características de uma determinada população, fenômeno ou o estabelecimento que esteja envolvido ou tenha relações entre variáveis do mesmo seguimento. Envolvendo o uso de técnicas padronizadas direcionadas a coleta de dados, utilizando questionários e observação sistemática ao fenômeno em pesquisa (GIL AC, 1999).

Foram disponibilizados recursos entre o monitor e aos alunos como o contato pelas redes sociais (WhatsApp, E-mail) com intuito de que suas dúvidas e problemas fossem rapidamente solucionadas. Dessa forma, os assuntos eram enviando com antecedência, dando tempo para os alunos revisarem para próxima aula, solucionando as dúvidas maior facilidade.

O programa de monitoria dispõe da carga horaria de 15 horas mensais, durante o período de 06 meses. As atividades acadêmicas estavam relacionadas a supervisão, orientação e auxílio de diversas formas aos alunos e ao professor, garantindo a troca de conhecimento, contato com o todo e suas partes, através de aulas teóricas, práticas, revisões e auxilio na aplicação das provas.

As atividades de monitoria foram desenvolvidas em dois momentos, sendo o primeiro momento voltado para parte teórica, e o segundo para realização de práticas laboratoriais, como técnicas e procedimentos de enfermagem. Uma vez que, os dois momentos são de extrema importância para que o aprendizado possa ser compreendido e fixado de maneira correta, possibilitando assim a revisão e aprendizagem dos conteúdos ministrados anteriormente.

REAEnf/EJNC | Vol. 6 | e5685 | DOI: https://doi.org/10.25248/REAenf.e5685.2020 Página 3 de 6 
No primeiro momento, as aulas teóricas estavam direcionadas principalmente em dar auxílio ao professor em sala de aula, desenvolvendo diversas atividades como auxiliar na aplicação e correção das provas, realização da frequência, ajudar alunos com dúvidas sobre os assuntos ministrados em aula, e principalmente em questões relacionadas a seminários e trabalhos acadêmicos. Uma vez que, a grade curricular da disciplina e bastante extensa e requer uma atenção maior dos discentes para os conteúdos ministrados. Houve também apresentações em forma de seminários realizado pelos alunos como método de avaliação parcial (Quadro 1).

Quadro 1 - Seminários apresentados pelos alunos em sala de aula.

\begin{tabular}{|c|}
\hline Apresentações \\
\hline Cuidados com paciente pós-morte; \\
\hline Aplicação de calor e frio; \\
\hline Escala de Dor; \\
\hline Banho no leito; \\
\hline Higiene intima; \\
\hline Higiene oral; \\
\hline Tricotomia. \\
\hline
\end{tabular}

Fonte: Barbosa IEB, et al., 2020.

No segundo momento, relativo as aulas práticas iniciaram-se no laboratório de suporte básico de vida, com a realização de práticas que foram ministradas em sala de forma teórica. As atividades foram desenvolvidas primeiramente pela professora, que em sequência explicava os detalhes da técnica e depois solicitava que os alunos as realizassem. Em seguida, houve uma mesa redonda para verificar quais as possíveis dificuldades apresentadas pelos alunos e tentar solucionar os problemas (Quadro 2).

Quadro 2 - Procedimentos realizados na monitoria.

\begin{tabular}{|c|}
\hline Práticas de enfermagem realizadas no laboratório \\
\hline Higienização das mãos; \\
\hline Aferição de sinais vitais; \\
\hline Calçamento de luvas; \\
\hline Mudança de decúbito; \\
\hline Cálculo, diluição e administracao de medicamentos, Punção venosa; \\
\hline Equipamentos de Proteção Individuais (EPIs); \\
\hline Passagem de cateter, nasogástrico e nasoenteral; \\
\hline Transporte de paciente, Restrição no leito; \\
\hline Sondagem vesical de demora e de alívio; \\
\hline Exames físicos, Aferição de PA, ausculta Cardíaca e Pulmonar. \\
\hline
\end{tabular}

Fonte: Barbosa IEB, et al., 2020.

O laboratório é um ambiente destinado ao aprendizado e revisão das técnicas que foram ministrados em sala de aula de forma teórica, onde o aluno simula a realização de procedimentos e técnicas de enfermagem, aperfeiçoando suas habilidades antes de entrar em contato com o paciente diretamente, sendo considerado assim, um espaço de produção, reprodução e reflexão de conhecimento, pois os procedimentos ali são realizados iram fazer parte da sua futura vida como um profissional de enfermagem (CARVALHO LD, et al., 2012).

\section{DISCUSSÃO}

O exercício da monitoria oportunizou uma nova gama de aquisição de conhecimento teórico-prático, além de instigar o interesse pela prática do profissional docente como futura profissão, uma vez que fomenta a formação integrada do aluno nas atividades vinculadas ao ensino, pesquisa e extensão que são disponibilizados dentre de uma instituição, seja ela pública ou privada (SILVA JDS, et al., 2012). 
O monitor, durante o processo de monitoria acadêmica, desenvolve uma análise crítica sobre o processo de ensino e aprendizagem. Uma vez que, ao entrar em contato com diferentes metodologias que são desenvolvidas pelos professores, e possível, que o aluno-monitor possa elaborar sua própria metodologia, contribuindo assim, não somente para novos processos de monitorias, mas também para que possa surgir o interesse à docência como futura profissão. Sendo que, a monitoria e o principal processo contribuinte para que esse aluno-monitor venha desenvolver seus conhecimentos e optar pela carreira docente durante 0 processo de graduação. (CARVALHO LD, et al., 2012).

Neste estudo, entende-se a importância monitoria acadêmica como uma da principal forma de apoio ao ensino/aprendizagem dos discentes. Uma vez que, os estudantes que já concluíram a disciplina contribuem ainda mais para o processo de apropriação do conhecimento de seus colegas que ainda estão cursando. Portanto, tem por finalidade auxiliar o professor e os alunos, usando as metodologias ativas no processo de aprendizagem. Tendo em vista que, esse monitor irá colaborar no aprimoramento dos estudantes quanto as técnicas de enfermagem e práticas laboratoriais (TOSTES MFP, et al., 2017).

A monitoria acontece principalmente na sala de aula, porém pode também acontecer em locais distintos como em laboratórios, na biblioteca, em residência, e até mesmo congressos acadêmicos, já o tempo, ele pode ser organizado para realização de atividades em horário de aula, fora da classe, ou ambas as situações, isso irá depender do tipo de atividade que será desenvolvida e as pessoas que iram participam desse projeto, o ambiente deve propiciar a livre comunicação entre todos e expressão de ideias e sentimentos, bem como a cooperação (NATÁRIO EG e SANTOS AA, 2010).

Em relação aos conteúdos que foram ministrados para os alunos, tanto nas aulas práticas como nas teóricas. Foi percebido que uma parte dos alunos apresentaram dificuldades iniciais em relação há algumas aulas práticas como aplicação de medicamentos e sondagem, uma vez que requer uma atenção maior dos discentes para essas técnicas. Porém, no decorrer da disciplina Semiologia e Semiotécnica, foi possível perceber que os alunos desenvolveram habilidades na execução de procedimentos necessários à assistência de enfermagem. Visto que, a ementa desta disciplina proporciona uma assistência de enfermagem ao indivíduo, família e comunidade no atendimento de suas necessidades humanas básicas.

A monitoria acadêmica e uma das principais práticas contribuintes para docência como futura profissão, pois o aluno-monitor adquire novas habilidades e trabalha sua postura diante de determinadas situações no decorrer da graduação, no entanto, essa atividade ainda precisa ser aprimorada para atender ao objetivo de se preparar corretamente esses acadêmicos para a vida docente (SILVA RN e BELO MLM, 2012).

A prática da monitoria-acadêmica é uma chance para o aluno-monitor tem de ampliar habilidades inerentes e conhecimentos adquiridos sobre à docência, aprofundando seus conhecimentos na área especifica e contribuir para a melhor aprendizagem de novos alunos monitorados (CARDOSO MC e RAÚJO RP, 2008).

Desse modo, é incrível acompanhar e observar o crescimento dos alunos. Tendo em vista que, a disciplina de semiologia e semiotécnica tem uma grade curricular muito extensa, e poder acompanhar o desenvolvimento dos alunos sabendo que eles estão realmente dispostos a trilhar seus caminhos na área da saúde e bastante satisfatório. Diante disso, durante a de construção do conhecimento, podemos dizer que, no decorrer desse processo, que envolve os docentes e monitores, surgem novas ideias, com o objetivo de melhorar ainda mais a atividade que é desenvolvida com os alunos, complementando assim as aulas, com a finalidade de apresentar e desenvolver uma educação de qualidade em prol de todos.

Assim, a prática da monitoria acadêmica contribui de forma grandiosa no processo de ensinoaprendizagem desses alunos, proporcionando a qualificação e revisão dos assuntos ministrados em aula, havendo a oportunidade de obterem uma nova visão sobre a docência. Uma vez que, ao finalizar o processo de monitoria os decentes se tornam capazes de intensificar a relação entre os discentes matriculados na disciplina e o docente-instituição (SANTOS SH e BATISTA SH, 2015).

De acordo com as informações apresentadas, conclui-se que a experiência na monitoria de semiologia e semiotécnica, foram de suma importância para o desenvolvimento pessoal e profissional. Pois, possibilitou uma nova oportunidade na vida acadêmica, tanto pela possibilidade de rever os conteúdos ministrados em

REAEnf/EJNC | Vol. 6 | e5685 | DOI: https://doi.org/10.25248/REAenf.e5685.2020 Página 5 de 6 
aula, como também para adquirir novos conhecimentos, visando a melhoria da qualidade do ensinoaprendizagem, proporcionando crescimento e aprofundamento nos conhecimentos relacionados as disciplinas.

\section{REFERÊNCIAS}

1. ALMEIDA RS, et al. Contribuições da monitoria em elementos de anatomia para a formação acadêmica do aluno de psicologia: um relato de experiência. Cadernos de Graduação, 2016; 3(3): 169-180.

2. CARDOSO MC, ARAÚJO RP. Monitoria acadêmica: relato de experiência em disciplina aplicada da Terapia Ocupacional. 2008; 16(1), 53-57.

3. CARVALHO LD, et al. Monitoria em semiologia e semiotécnica para a enfermagem: um relato de experiência. Rev Enferm UFSM, 2012; 2(2).

4. FERNANDES NC, et al. Monitoria acadêmica e o cuidado da pessoa com estomia: relato de experiência. Revista Mineira de Enfermagem, Belo Horizonte, 2015; 19(2), 238-241.

5. FRISON LM. Monitoria: uma modalidade de ensino que potencializa a aprendizagem colaborativa e autorregulada. Pró-Posições. 2016; 27(1), 133-153.

6. GIL AC. Como elaborar projetos de pesquisa. São Paulo: Atlas, 1991.

7. GUEDES DH, et al. J. R. Iniciação à docência com a monitoria, contribuindo para a melhoria do ensino no curso de Zootecnia e Agronomia no período 2007/1.

8. GURGEL SS, et al. Jogos educativos: recursos didáticos utilizados na monitoria de educação em saúde. Rev Min Enferm. 2017; 21 : e-1016.

9. MELO GSM, et al. Semiologia e Semiotécnica da enfermagem: avaliação dos conhecimentos de graduandos sobre procedimentos. Revista brasileira da enfermagem, Brasília, 2016; 70(2).

10. NATÁRIO EG, SANTOS AA. Programa de monitores para o ensino superior. Campinas: 2010. Guedes, M. L. (1998). Monitoria: uma questão curricular e pedagógica (Série acadêmica, n.9, pp.3-9) Campinas: PUC-Campinas.

11. SANTOS AR. A metodologia cientifica: Construção do conhecimento - 8ª̣d. São Paulo: Editora, 2015.

12. SANTOS GM, BATISTA SH. Monitoria acadêmica em formação em/para saúde: desafios e possibilidades no âmbito de um currículo interprofissional em saúde. ABCS Health Sciences, São Paulo, 2015; 40(3), 203-207.

13. SILVA JDS, et al. Monitoria acadêmica na disciplina enfermagem em atenção à saúde da mulher: um relato de experiência. 2012.

14. SILVA RN, BELO LM. Experiências e reflexões de monitoria: contribuição ao ensino aprendizagem. Scientia plena, 2012; 8(7).

15. STEINDORFF G, et al. Monitoria acadêmica no componente curricular de Semiotécnica em Enfermagem: Relato de experiência. Anais do Salão Internacional de Ensino, Pesquisa e Extensão, 2016.

16. TOSTES MFP, et al. Habilidades e dificuldades técnico científicas dos acadêmicos de enfermagem durante a monitoria de semiotécnica. Rev Enferm UFPE online. 2017;11(5):1819-25. 